\title{
Renal Function in Nucleos(t)ide Analog-Treated Patients With Chronic Hepatitis B: A Systematic Literature Review and Network Meta-Analysis
}

\author{
Henry L. Y. Chan · Javed Shaikh · Subhajit Gupta · Kamal Hamed
}

Received: March 15, 2016 / Published online: May 4, 2016

(C) The Author(s) 2016. This article is published with open access at Springerlink.com

\begin{abstract}
Introduction: Renal safety is an important factor in selecting the most appropriate nucleos(t)ide analog (NA) treatment for patients with chronic hepatitis B (CHB). This systematic literature review and network meta-analysis aimed to assess renal function associated with telbivudine treatment compared to other NAs in patients with CHB.
\end{abstract}

Methods: A systematic literature search via Medline, Medline In-Process, Embase, and the

Enhanced Content To view enhanced content for this article go to www.medengine.com/Redeem/F2C4F0605 0605C5E.

Electronic supplementary material The online version of this article (doi:10.1007/s12325-016-0337-2) contains supplementary material, which is available to authorized users.

H. L. Y. Chan

Department of Medicine and Therapeutics and

Institute of Digestive Disease, The Chinese

University of Hong Kong, Hong Kong SAR, China

J. Shaikh · S. Gupta

Novartis Healthcare Pvt. Ltd., Hyderabad, India

K. Hamed (ه)

Novartis Pharmaceuticals Corporation,

East Hanover, NJ, USA

e-mail: kamal.hamed@novartis.com
Cochrane library for publications of randomized controlled trials and observational studies was conducted. Network meta-analysis was performed to compare renal function with telbivudine treatment versus other NAs after 1 year of therapy.

Results: Overall, 40 (six randomized controlled and 34 observational) studies were included for review. Telbivudine consistently showed an improvement in renal function as measured by an estimated glomerular filtration rate (eGFR) over various time points regardless of the method of measurement. Changes in eGFR $(\mathrm{mL} / \mathrm{min})$ from baseline and corresponding 95\% credible intervals with various NAs were as follows: monotherapies (telbivudine: 7.78 [6.91, 8.65], entecavir: $-1.07 \quad[-4.80,2.62]$, lamivudine: -6.08 [-13.35, 1.15], tenofovir: -9.53 [-14.31, -4.89]) and combination therapies (telbivudine + adefovir: 8.37 [-34.00, 50.34], telbivudine + tenofovir: $8.29 \quad[-0.05,16.64]$, entecavir + adefovir: $\quad 4.15 \quad[-38.55,46.37]$, telbivudine + lamivudine: 0.51 [-11.77, 12.96], and lamivudine + adefovir: $\quad-0.39 \quad[-42.48$, 41.21]). At 1 year, the change in eGFR from baseline was significantly higher with telbivudine compared to other NAs. 
Conclusion: The systematic literature review and network meta-analysis provide evidence that telbivudine is associated with significant improvement in renal function in patients with $\mathrm{CHB}$, either alone or in combination with other NAs.

Funding: Novartis Pharma AG.

Keywords: Chronic hepatitis B; eGFR; Hepatology; Infectious disease; Nucleos(t)ide analogs; Renal function; Telbivudine

\section{INTRODUCTION}

Hepatitis B virus (HBV) infection is a major global public health concern. Evidence from available data suggest that an estimated 240 million individuals are chronically infected with HBV worldwide, and approximately one million die from chronic hepatitis B (CHB)-related diseases every year $[1,2]$. Previous studies have described an association between $\mathrm{CHB}$ and chronic kidney disease (CKD) [3-6]. Nearly $15-30 \%$ of patients with CHB have baseline renal dysfunction or comorbidities associated with CKD, such as diabetes and hypertension. Findings from previous studies have implicated glomerular diseases such as membranous nephropathy and mesangiocapillary glomerulonephritis as the underlying causes of renal dysfunction caused by HBV [7, 8]. Renal dysfunction, marked by a decrease in estimated glomerular filtration rate (eGFR), is frequently observed in patients with compensated CHB. Nucleos(t)ide analogs (NAs), the mainstay of $\mathrm{CHB}$ therapy, are primarily eliminated unchanged through renal route [9]. Adefovir and tenofovir have been associated with nephrotoxicity related to the accumulation of nucleotide metabolites in renal tubular cells [10]. An observational cohort study conducted in patients with CHB from two German centers concluded that treatment of HBV monoinfection with adefovir $(n=32)$, entecavir $(n=32)$, lamivudine $(n=36)$, or tenofovir $(n=37)$ resulted in a mild decrease in renal function [11-13]. Therefore, renal safety is an important factor in selecting the most appropriate NA treatment for patients with $\mathrm{CHB}$, particularly in those who are at risk for renal impairment. Recent literature suggests that telbivudine is associated with improvement in renal function in patients with CHB. However, there is a lack of systematic evidence base regarding the comparison of renal function associated with NAs. Hence, it is important to collate all available evidence and summarize the data regarding the effect of various NAs on renal function in patients with $\mathrm{CHB}$, either as monotherapy or combination therapy. The aim of the current systematic literature review (SLR) and a network meta-analysis (NMA) is to assess renal function associated with telbivudine treatment compared to other NAs in patients with CHB.

\section{METHODS}

This SLR followed a standard, systematic review methodology endorsed by the Cochrane Collaboration [14] and the National Institute for Health and Care Excellence (NICE) in the UK [15]. The SLR was conducted and reported in accordance with the Preferred Reporting Items for Systematic Reviews and Meta-Analyses (PRISMA) guidelines [16].

\section{Inclusion and Exclusion Criteria}

\section{Inclusion Criteria}

All randomized controlled trials (RCTs) and observational studies published in the English 
language, conducted in patients with $\mathrm{CHB}$ regardless of their hepatitis $B$ envelope antigen (HBeAg) status, were included. Studies evaluating interventions/comparators (either monotherapy or combination therapies) such as adefovir, entecavir, lamivudine, telbivudine, tenofovir, and placebo with reported eGFR outcomes (either absolute change or percentage improvement from baseline) were included.

\section{Exclusion Criteria}

Animal and in vitro studies; studies in patients co-infected with human immunodeficiency virus, hepatitis C virus, or hepatitis D virus; and studies that did not report interventions, comparators, and outcomes of interest were excluded. In addition, review articles, editorials, case reports, case series, and economic evaluations were excluded.

\section{Search Strategy}

Comprehensive literature searches were conducted by searching electronic databases (Medline, Medline In-Process, Embase, and the Cochrane Library) from the inception of each database to July 2015. The multistring search strategy was based on a combination of medical subject headings (MeSH) and keywords including "adefovir", "entecavir", "lamivudine", "telbivudine", "tenofovir", "chronic hepatitis B", "hepatitis B", "chronic kidney disease", "CKD", "glomerular filtration rate", and "eGFR". Reference lists of any relevant studies, recent systematic reviews, or meta-analyses were also searched for any additional studies to add to the evidence base.

\section{First-Level Screening of Citations}

All the publications retrieved from the literature search were screened based on the title and abstract provided with each citation. The inclusion/exclusion criteria were uniformly applied across all the publications. Two independent reviewers screened the retrieved abstracts, and a third independent reviewer reconciled any discrepancies between them. Publications that did not meet the eligibility criteria were excluded, and the reasons for exclusion were documented. Subsequently, full-text copies of all the publications that met the eligibility criteria were downloaded.

\section{Second-Level Screening of Citations}

The inclusion and exclusion criteria were uniformly applied across all the full-text publications. The same two reviewers screened all the manuscripts, and the third independent reviewer reconciled any discrepancies between them. Publications that did not meet the eligibility criteria were excluded, and the reasons for exclusion were documented. Publications that met the eligibility criteria were subjected to data extraction.

\section{Data Extraction}

The same reviewers extracted data independently using a prespecified data extraction template, and as before, the third independent reviewer resolved any discrepancies. Data were extracted from various information sources in a study, such as objectives, methods, inclusion and exclusion criteria, baseline demographic and clinical characteristics, interventions, efficacy 
outcomes, and conclusions. Studies with multiple publications were linked to one another and extracted as a single study.

\section{Critical Appraisal (Quality Assessment)}

Each included study was assessed for methodological quality (internal and external validity). RCTs that met the eligibility criteria for review were critically appraised for quality based on the NICE recommendations [15]. All included observational studies were critically appraised for quality based on the Downs and Black checklist [17].

\section{Assessment of Endpoints}

The primary endpoint of the analysis was change in eGFR from baseline. Most of the studies reported the renal function outcomes at 48-52 weeks. There were few studies reporting renal function outcomes at 2-year follow-up. Hence, the study endpoints were analyzed at a time point of 1 year (48-52 weeks). For studies that did not report change in eGFR from baseline, the change was calculated based on the reported baseline and endpoint eGFR values.

\section{Network Meta-Analysis}

The NMA was performed using statistical methods to combine data from various studies in order to obtain a coherent picture of treatment outcomes and compare various treatment options. The statistical models for conducting NMA relate the underlying outcomes to the effects of treatment and any other factors (covariates). The models used to conduct this NMA were derived from the Report of the International Society for Pharmacoeconomics and Outcomes Research (ISPOR) Task Force on Indirect Treatment
Comparisons Good Research Practices: Part 2 [18] and the NICE Technical Support Document 2 (TSD2) [19]. For the purpose of the present analysis, Bayesian models were used. Furthermore, in order to assess the inconsistencies in the evidence networks, we compared a standard network consistency model with an "inconsistency" or unrelated mean effects model. According to the NICE Decision Support Unit (DSU) guidelines, this methodology is more efficient compared to existing methodologies to detect inconsistencies [20]. The statistical software $\mathrm{R}$ (version 3.2.2, $\mathrm{R}$ Foundation for Statistical Computing, Vienna, Austria) was used for analysis. An integrated graphical user interface (GUI) for R, Rstudio (version 0.98.945), was used to run all the R scripts. The package R2WinBUGS was used to retrieve WinBUGS 1.4 from $\mathrm{R}$ in order to run the Bayesian Markov Chain Monte Carlo (MCMC) algorithm. A feasibility check was performed to conduct the NMA, and only non-RCTs were found to be eligible. All non-RCTs were assumed comparable in terms of study design, and for studies with missing standard error (SE) values, SE was assumed to be $10 \%$ of the mean change in eGFR from baseline.

\section{Compliance with Ethics Guidelines}

This article is based on previously conducted studies and does not involve any new studies of human or animal subjects performed by any of the authors.

\section{RESULTS}

\section{Search Results}

The results of the literature search are summarized in Fig. 1. The search of the 


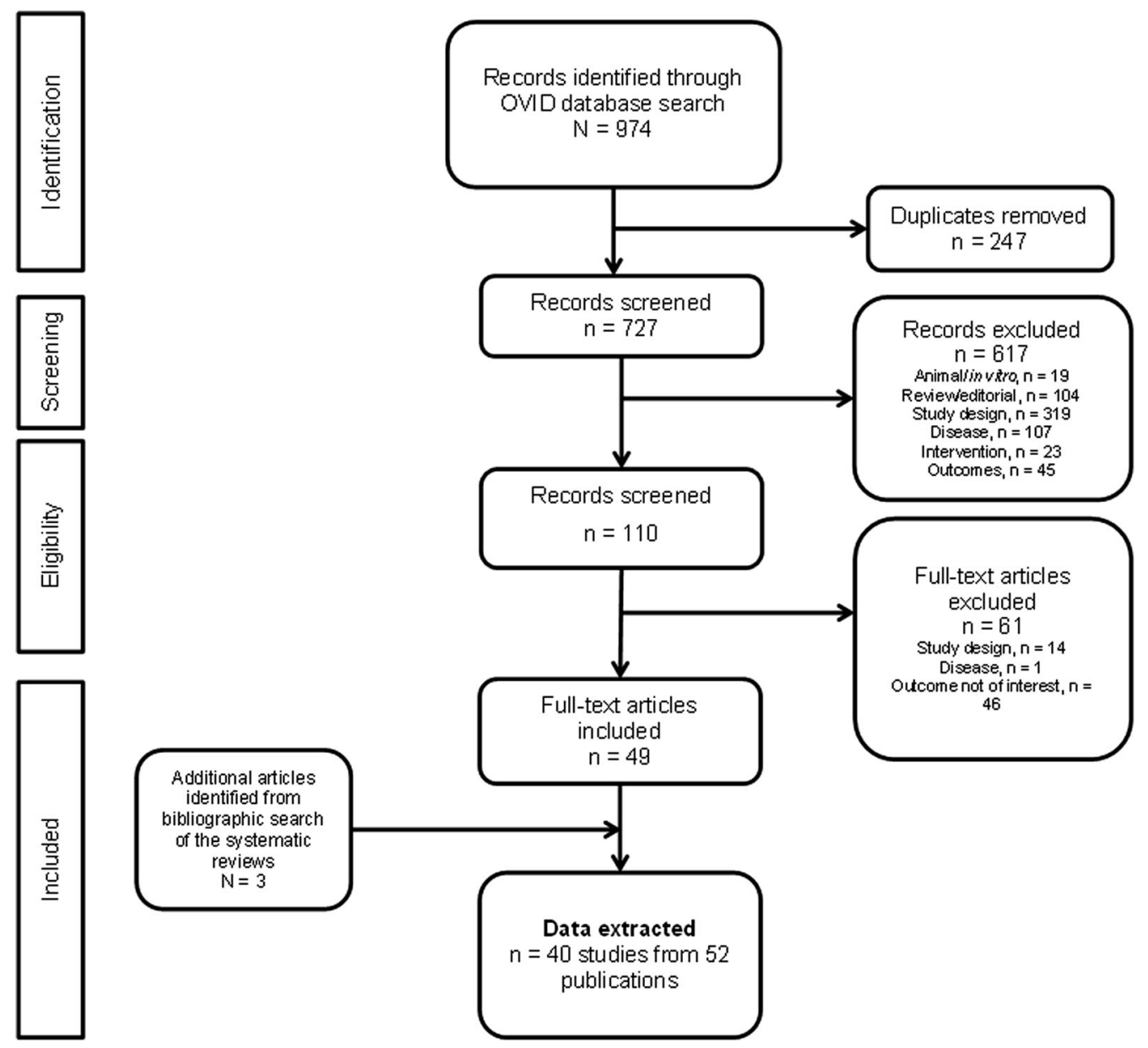

Fig. 1 Flow diagram of the literature search

literature databases yielded 974 publications. Of these, 247 were duplicates due to an overlap of records across the searched databases. Following the initial screening of these citations, 110 potentially relevant publications were identified and 49 of them were included after a more detailed evaluation. Three publications were additionally identified through a bibliographic search of the relevant systematic reviews, yielding 40 studies from 52 publications for synthesis of evidence.

\section{Study Characteristics}

Of the 40 studies included, 20 were full-text publications and 20 were conference abstracts. In total, six RCTs and 34 observational studies were included (17 retrospective studies, 12 prospective studies, three non-RCTs, and one case-control study and cross-sectional study each). Of the 40 included studies, 35 had an active control group, and in the remaining five studies, NAs were compared with untreated 
controls. Entecavir, telbivudine, and tenofovir were the most commonly reported treatments. Thirteen of the 40 studies were multicenter studies, whereas 16 studies had been conducted at a single center. In the remaining 11 studies, the study location was unclear. Overall, 90\% $(36 / 40)$ of the studies were conducted in populations with mixed HBeAg status, whereas only three studies [21-23] enrolled HBeAg-negative patients and one study [24] enrolled HBeAg-positive patients. The mean age among the included studies ranged from 34 [25] to 55 [26] years. Approximately 15\% (6/40) of the included studies enrolled over $70 \%$ of the male population. Modification of Diet in Renal Disease (MDRD) was the most commonly used equation to measure eGFR among the included studies (see Table S1 in the online supplementary material).

\section{Critical Appraisal (Quality Assessment)}

Of the six RCTs that exhibited similarities among treatment groups in terms of baseline characteristics, two studies reported an adequate method of allocation concealment and two reported an adequate method of blinding for patients and caregivers. An adequate method of statistical analysis was reported in three studies. For the observational studies, the majority of relevant information was missing from the conference abstracts; the Downs and Black total scores ranged from 2 [27] to 18 [28]. Overall, the methodological quality of the included studies was adequate.

\section{Trends for Changes in eGFR over Different Time Points}

Figure 2 shows the changes in eGFR over different time points across the included studies for entecavir, lamivudine, telbivudine, and tenofovir. The plots clearly indicate that telbivudine consistently showed an increase in eGFR over different time points regardless of the method used to measure eGFR. Four studies reported eGFR with lamivudine at different time points. A retrospective study comparing long-term renal function with different NAs [29] reported eGFR in 77 lamivudine-treated patients according to the MDRD method. The baseline eGFR was $86.58 \mathrm{~mL} / \mathrm{min} / 1.73 \mathrm{~m}^{2}$, and the mean [standard deviation (SD)] change from baseline at 52 weeks was -3.55 (19.13). In an RCT comparing the safety and efficacy of telbivudine and lamivudine, patients who received lamivudine showed a steady decline in renal function, as evidenced by the evolution of serum creatinine and eGFR [estimated using the MDRD and Chronic Kidney Disease Epidemiology Collaboration (CKD-EPI) methods] [30]. Ten studies reported eGFR with telbivudine treatment at different time points. According to these studies, telbivudine improved renal function. A study by Gane et al. [31] reported eGFRs calculated using the MDRD, CKD-EPI, and Cockcroft-Gault (CG) methods, and indicated improved renal function in telbivudine-treated patients from the 2-year GLOBE study. Based on the MDRD equation, the mean eGFR had increased by $8.5 \%$ in telbivudine-treated patients. Improved renal function was maintained for 4-6 years. In addition, increased eGFR with telbivudine treatment was observed in patients at an increased risk for renal impairment: patients with a baseline eGFR of $60-89 \mathrm{~mL} / \mathrm{min} / 1.73 \mathrm{~m}^{2}$ $(+17.2 \%)$, those aged $>50$ years $(+11.4 \%)$, and those with liver fibrosis/cirrhosis $(+7.2 \%$ for patients with Ishak fibrosis score of 5-6). In decompensated patients with a high renal risk, eGFR improved with telbivudine $(+2 \%)$. In total, eight studies had reported eGFR with entecavir at different time points. A study [26] 

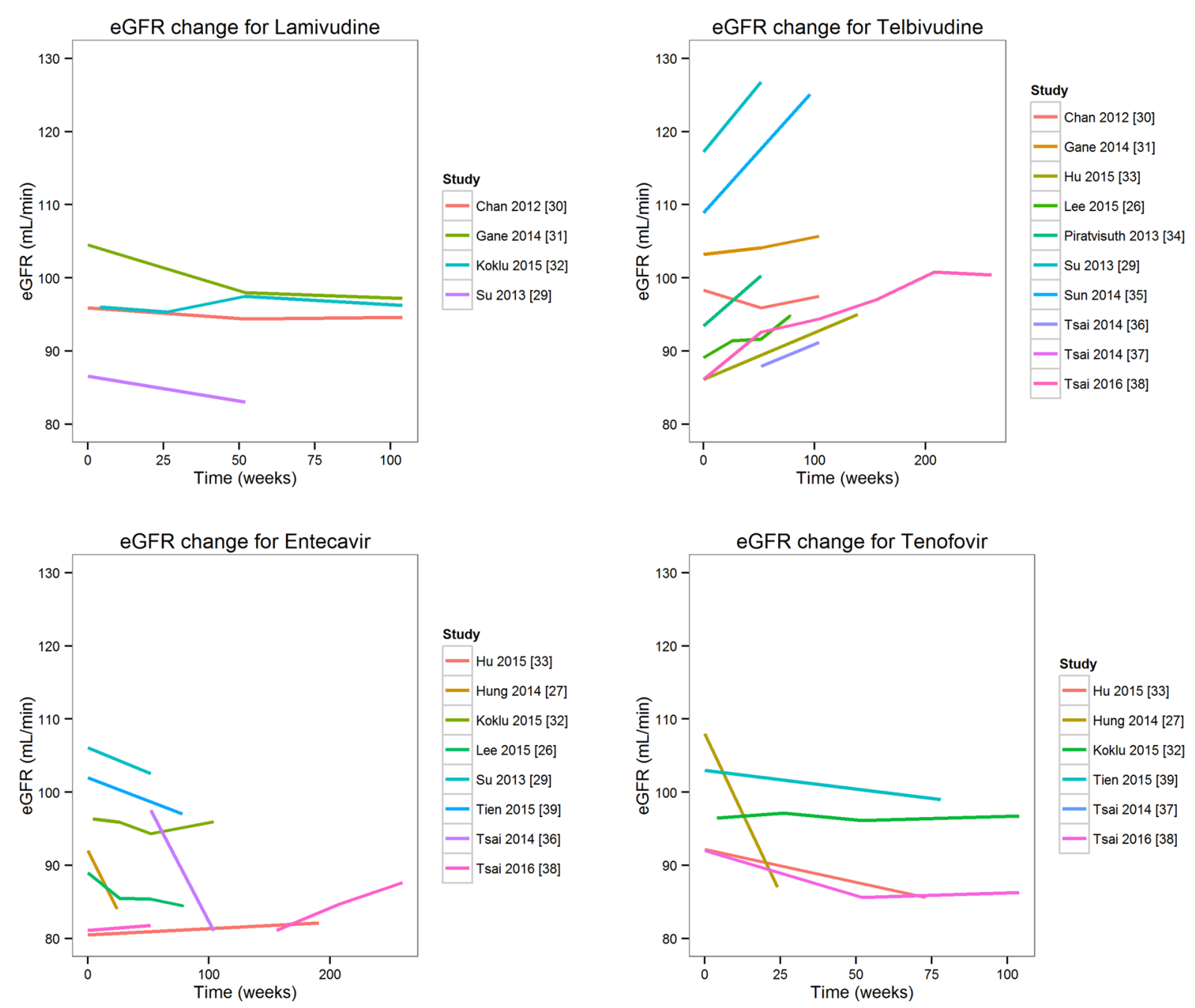

Fig. 2 Trends for eGFR changes over different time points. $e G F R$ estimated glomerular filtration rate

conducted in entecavir-treated patients reported a mean decline in eGFR by $4.1 \%$ at month 18 compared with baseline.

In a retrospective study [38] conducted in patients with baseline stage II CKD (eGFR, $60-90 \mathrm{~mL} / \mathrm{min} / 1.73 \mathrm{~m}^{2}$ ), entecavir showed improvements in renal function from 75.8 to $82.5 \mathrm{~mL} / \mathrm{min} / 1.73 \mathrm{~m}^{2}$. Only a few studies had reported eGFR with adefovir at different time points; hence, a trend diagram could not be constructed. However, eGFR decreased persistently with adefovir treatment. The annual eGFR decline from baseline was $5.62 \mathrm{~mL} / \mathrm{min}$ (first year), $11.26 \mathrm{~mL} / \mathrm{min}$ (second year; $P=0.0008$ vs. baseline), and $13.72 \mathrm{~mL} / \mathrm{min}$ (third year; $P=0.0005$ vs. baseline) [40].

Figure 3 and Table S2 in the online supplementary material display the trends in eGFR changes from baseline at 1 year for different NAs. Both clearly show an increase in eGFR from baseline with telbivudine treatment, alone with in combination with other NAs, across all the included studies with varying sample sizes. 


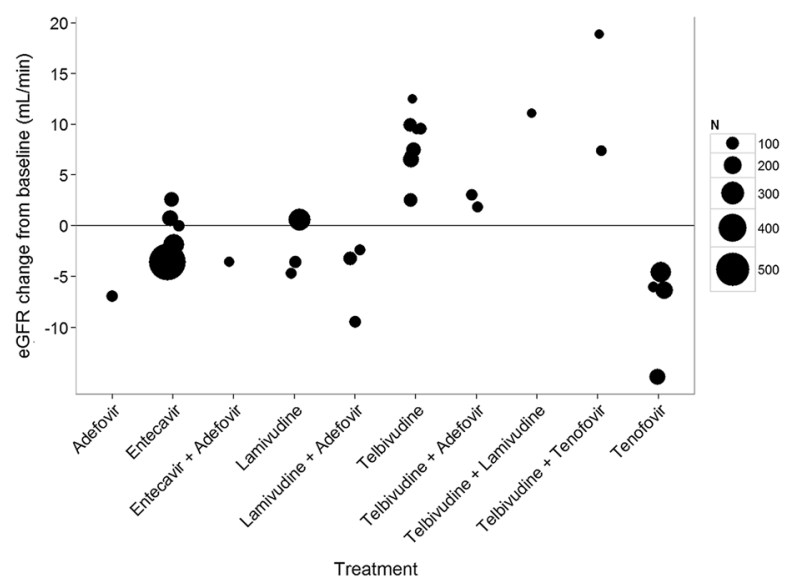

Fig. 3 Trends for eGFR changes from baseline at 1 year. The size of the bubble corresponds to the sample size for corresponding treatment. $e G F R$ estimated glomerular filtration rate

\section{Hurdles and Assumptions for NMA}

A feasibility check was performed to ascertain the possibility of conducting an NMA with available data. The included studies were widely heterogeneous, and considering specific assumptions, an NMA was possible only in the non-RCTs. For eGFR changes from baseline at 1 year in the non-RCTs, it was possible to construct a network diagram for available evidence. The assumptions used to attempt the NMA were as follows: all non-RCTs were comparable in terms of baseline characteristics and missing SE was computed to be $10 \%$ of the mean change in eGFR from baseline. For the purpose of analysis, the eGFR values from different equations were analyzed together.

\section{eGFR Changes from Baseline at 1 Year (NMA Results)}

The network diagram (Fig. 4) shows the full network of evidence of treatment regimens for changes in eGFR from baseline at the 1-year time point. Overall, NMA was feasible for 12 non-RCTs.
Figure 5 shows the NMA results for NAs. Changes in eGFR ( $\mathrm{mL} / \mathrm{min})$ from baseline and corresponding $95 \%$ credible intervals for different NAs were as follows: monotherapies (telbivudine: 7.78 [6.91, 8.65], entecavir: -1.07 [-4.80, 2.62], lamivudine: -6.08 [-13.35, 1.15], and tenofovir: $-9.53[-14.31,-4.89])$ and combination therapies (telbivudine + adefovir: 8.37 [-34.00, 50.34], telbivudine + tenofovir: 8.29 [-0.05, 16.64], entecavir + adefovir: 4.15 [-38.55, 46.37], telbivudine + lamivudine: 0.51 $[-11.77,12.96]$, and lamivudine + adefovir: -0.39 [-42.48, 41.21]). Therefore, results from the current NMA strongly suggested that telbivudine, either as monotherapy or in combination with other NAs, showed improvements in positive values for eGFR change from baseline. The other NAs consistently resulted in negative values for eGFR change from baseline.

\section{DISCUSSION}

The present study assessed renal function associated with telbivudine treatment compared to other NAs in patients with CHB. Telbivudine consistently showed an increase in eGFR across various time points, regardless of the method used to measure eGFR. The NMA results for changes in eGFR from baseline at 1 year suggested that compared with other NAs, telbivudine was associated with an increase in eGFR across all included studies with varying sample sizes. Initially, the study intended to analyze changes in eGFR from baseline at a 2-year time point. However, considering the limited number of studies reporting data at 2 years, construction of a network diagram for NMA was not feasible. Over the years, published literature has provided increasing evidence for the 


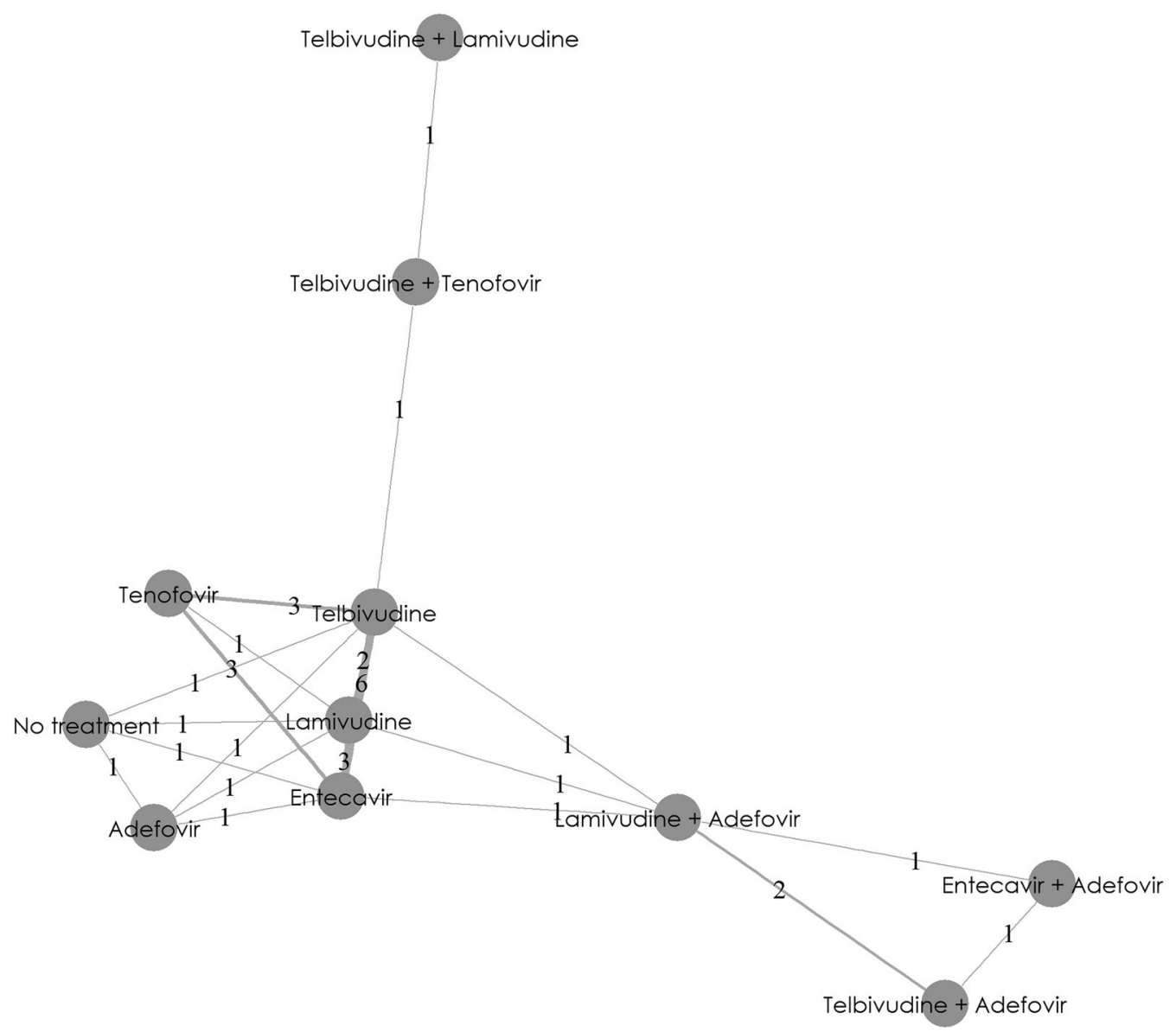

Fig. 4 eGFR changes from baseline at 1 year: network diagram. This network diagram provides a graphical summary of direct and indirect evidence available from trials. A node represents each treatment option. Lines connecting two nodes represent the direct evidence

renoprotective effects of telbivudine [26, 30, $31,38,40]$. The mechanism of eGFR improvement with telbivudine remains to be determined. Numerous studies have shown that the decline in eGFR associated with adefovir or tenofovir treatment is attributed to the activity of these drugs against human mitochondrial DNA (mtDNA) [34]. The proximal tubules are intrinsically susceptible to mitochondrial dysfunction because of limited anaerobic adenosine triphosphate (ATP)-generating capacity [41]. These NAs (adefovir and tenofovir) are primarily excreted comparing the treatments. The numbers between the lines represent the number of studies available for that particular treatment comparison. eGFR estimated glomerular filtration rate

in unchanged forms through urine; therefore, inhibition of renal $\gamma$-mtDNA may affect renal function [42].

A head-to-head cohort study compared the effects of various NAs on eGFR in Chinese patients with CHB [40]. The authors concluded that prolonged telbivudine therapy resulted in improvements in eGFR, whereas adefovir therapy was associated with a decrease in eGFR; moreover, lamivudine and entecavir did not significantly affect eGFR [40]. The European Association for the Study of the Liver (EASL) guidelines recommend that for patients at low 


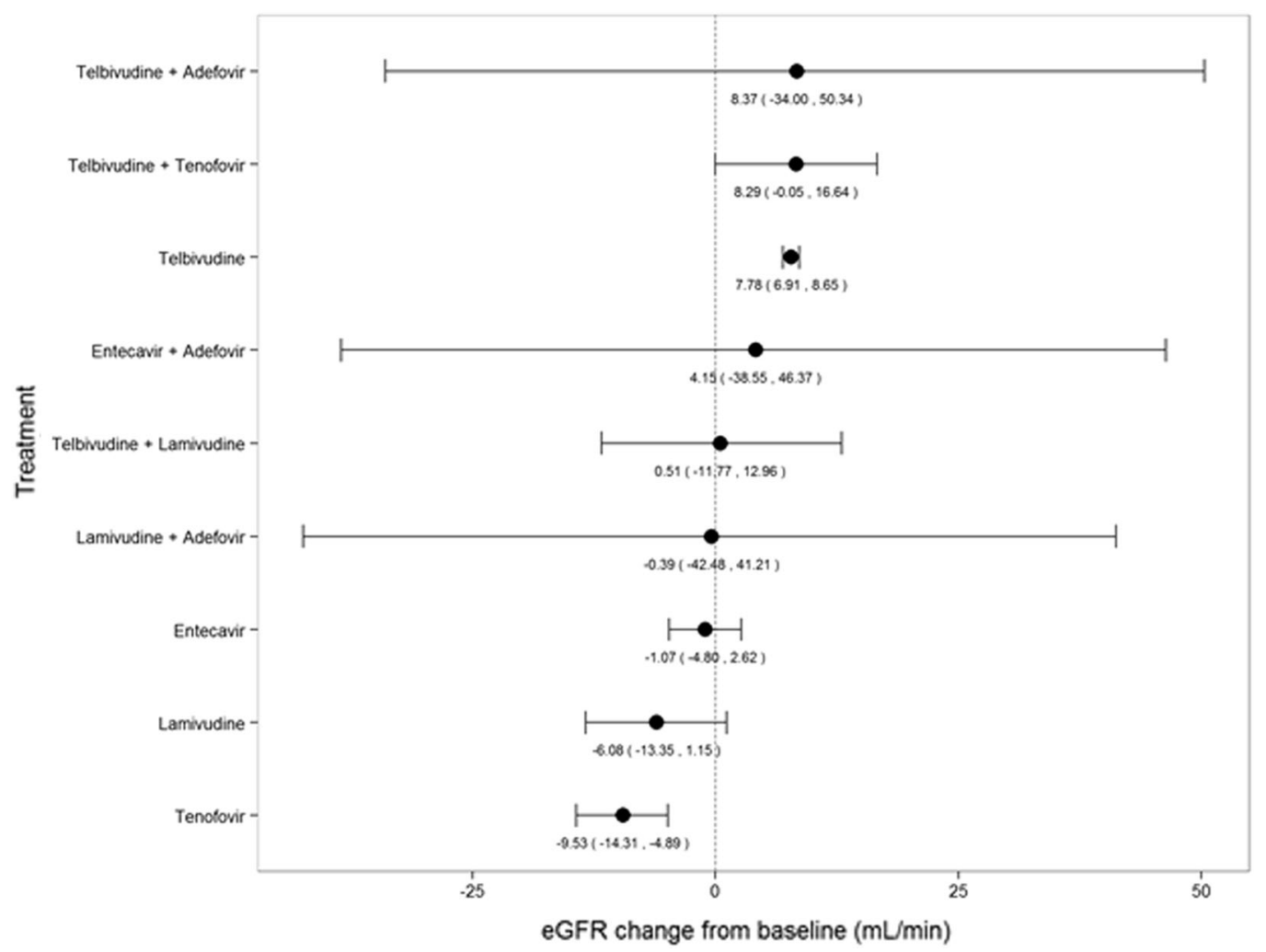

Fig. 5 eGFR changes ( $95 \%$ credible intervals) from baseline at 1 year: network meta-analysis results. eGFR estimated glomerular filtration rate

renal risk receiving long-term NA therapy, renal function should be monitored every 3 months during the first year and every 6 months thereafter if there is no worsening. For patients at a high renal risk, renal function should be monitored every month for the first 3 months, every 3 months until the end of the first year, and every 6 months thereafter if there is no worsening [43]. Gane et al. [31] have reported the renoprotective effects of telbivudine in a comprehensive analysis of renal function by using a telbivudine clinical trial database. This study evaluated renal function, focusing on the 2-year data from the GLOBE study. The authors showed that renal function, as assessed by the three equations for eGFR (CG, MDRD, and CKD-EPI), improved in patients who received telbivudine, whereas those who received lamivudine experienced a decline in renal function. Furthermore, an extension study of the GLOBE trial showed that even patients previously treated with lamivudine benefited from the renoprotective effects of telbivudine, with an additional increase in their eGFR of approximately $10 \%$. This study also reported that eGFR improvement was maintained during long-term (4-6 years) telbivudine treatment [31]. An open-label, 2-year extension study that assessed the safety and efficacy of long-term telbivudine treatment in 596 patients with $\mathrm{CHB}$ without genotypic resistance after 2 years of initial telbivudine treatment reported that telbivudine-treated patients showed a steady increase in eGFR over 2 years, which remained elevated for up to 4 years [44].

Only one meta-analysis has been published in the literature that evaluated the renal safety 
profile of adefovir in patients with CHB [45]. The authors concluded that based on currently available evidence, adefovir treatment is associated with an increased risk of renal dysfunction. However, this study was not an NMA, and it analyzed RCTs and single-arm studies differently and used composite outcomes to define renal dysfunction.

This SLR and NMA have both limitations and strengths. The main limitations of the NMA are the non-randomized designs and heterogeneity among the included studies. The NMA was feasible under the aforementioned assumptions, and the network diagram could be constructed with 12 non-RCTs. The NMA was not feasible with RCTs, because of the limited number of these studies. Another limitation is that half of the studies were conference abstracts. A sensitivity analysis was attempted by including only full-text publications (i.e., excluding conference abstracts). However, a network diagram for this sensitivity analysis was not feasible, because of the limited number of studies and available data. Similarly, separate analyses for patients with compensated versus decompensated cirrhosis, HBeAg-positive versus HBeAg-negative status, high versus low baseline HBV DNA level, and eGFR decline $\geq 20 \%$ versus $<20 \%$ were not possible. Moreover, eGFR values obtained by using different equations were analyzed together as there was not enough studies reporting use of every eGFR equation (MDRD was the most commonly used equation). The strengths of SLR and NMA techniques include the robustness of the methodology based on the methods and recommendations from the Cochrane handbook [14], and the NICE guidelines [15]. A well-defined protocol including comprehensive search strategy, eligibility criteria, and data points to be extracted was followed throughout the process. The analyses were performed using the Bayesian MCMC algorithm. Although the NMA was feasible only with non-RCTs, the data from RCTs consistently indicated that telbivudine was associated with improvement in renal function (see Table S3 in the online supplementary material).

Thus, results from both clinical trials and real-world studies strongly suggest that telbivudine is associated with improvements in renal function in patients with $\mathrm{CHB}$, either alone or in combination with other NAs, and these improvements were observed in patients with both compensated liver disease as well as decompensated cirrhosis. Further, the improvements in renal function were maintained for up to 6 years. Moreover, patients with mild renal impairment at baseline who were treated with telbivudine also experienced a consistent improvement in eGFR. Over the years, renal safety has emerged as an important treatment-related concern in NA-treated patients. Although viral resistance and adverse events due to NA treatment are key factors when selecting a particular therapy for $\mathrm{CHB}$, the current findings suggest that telbivudine offers a more favorable renal safety profile compared to other NAs, which may make it the drug of choice for patients at risk for renal dysfunction.

\section{CONCLUSION}

This SLR and NMA provide evidence that telbivudine is associated with a significant improvement in renal function (eGFR) in patients with $\mathrm{CHB}$, either alone or in combination with other NAs. 


\section{ACKNOWLEDGMENTS}

The article processing charges and the open access charge for this NMA were funded by Novartis Pharma AG. All named authors meet the International Committee of Medical Journal Editors (ICMJE) criteria for authorship of this manuscript, take responsibility for the integrity of the work as a whole, and have given final approval to the version to be published. Screening and data extraction were conducted by Ankush Taneja (Novartis Healthcare Pvt. Ltd.). Medical writing support was provided by Satabdi Chatterjee (Novartis Healthcare Pvt. Ltd.).

Disclosures. Henry L. Y. Chan received a research grant and honoraria for speaking at Novartis sponsored medical events. Javed Shaikh is an employee of Novartis Healthcare Pvt. Ltd. Subhajit Gupta is an employee of Novartis Healthcare Pvt. Ltd. Kamal Hamed is an employee of Novartis Pharmaceuticals Corporation.

Compliance with ethics guidelines. This article is based on previously conducted studies and does not involve any new studies of human or animal subjects performed by any of the authors.

Open Access. This article is distributed under the terms of the Creative Commons Attribution-NonCommercial 4.0 International License (http://creativecommons.org/licenses/ by-nc/4.0/), which permits any noncommercial use, distribution, and reproduction in any medium, provided you give appropriate credit to the original author(s) and the source, provide a link to the Creative Commons license, and indicate if changes were made.

\section{REFERENCES}

1. European Association for the Study of the Liver. EASL clinical practice guidelines: management of chronic hepatitis B virus infection. J Hepatol. 2012;57:167-85.

2. WHO fact sheet on Hepatitis B. 2015. http://www. who.int/mediacentre/factsheets/fs204/en/. Accessed Apr 18, 2016.

3. Combes B, Shorey J, Barrera A, et al. Glomerulonephritis with deposition of Australia antigen-antibody complexes in glomerular basement membrane. Lancet. 1971;2:234-7.

4. Chacko EC, Surrun SK, Mubarack Sani TP, Pappachan JM. Chronic viral hepatitis and chronic kidney disease. Postgrad Med J. 2010;86:486-92.

5. Bhimma R, Coovadia HM. Hepatitis B virus-associated nephropathy. Am J Nephrol. 2004;24:198-211.

6. Yi Z, Jie YW, Nan Z. The efficacy of anti-viral therapy on hepatitis $B$ virus-associated glomerulonephritis: a systematic review and meta-analysis. Ann Hepatol. 2011;10:165-73.

7. Lai KN, Li PK, Lui SF, et al. Membranous nephropathy related to hepatitis $B$ virus in adults. N Engl J Med. 1991;324:1457-63.

8. Appel G. Viral infections and the kidney: HIV, hepatitis B, and hepatitis C. Cleve Clin J Med. 2007;74:353-60.

9. Fontana RJ. Side effects of long-term oral antiviral therapy for hepatitis B. Hepatology. 2009;49(Suppl):S185-95.

10. Deray G, Buti M, Gane E, et al. Hepatitis B virus infection and the kidney: renal abnormalities in HBV patients, antiviral drugs handling, and specific follow-up. Adv Hepatol. 2015;2015:11. doi:10. $1155 / 2015 / 596829$ (article ID 596829).

11. Deterding K, Lampe N, Reijnders J, et al. Prevalence and severity of kidney dysfunction in patients with chronic hepatitis $B$ in Europe: data from the European VIRGIL cohort. In: Abstracts of the 46th Annual Meeting of the European Association for the Study of the Liver. Berlin: European Association for the Study of Liver; 2011. Abstract number 366.

12. Mauss S, Berger F, Filmann N, et al. Effect of HBV polymerase inhibitors on renal function in patients with chronic hepatitis B. J Hepatol. 2011;55:1235-40. 
13. Kim YJ, Cho HC, Sinn DH, et al. Frequency and risk factors of renal impairment during long-term adefovir dipivoxil treatment in chronic hepatitis B patients. J Gastroenterol Hepatol. 2012;27:306-12.

14. Higgins JPT, Green S, editors. Cochrane handbook for systematic reviews of interventions version 5.1.0 [updated March 2011]. The Cochrane Collaboration; 2011. http://www.handbook. cochrane.org. Accessed Apr 18, 2016

15. National Institute for Health and Care Excellence. Guide to the methods of technology appraisal. http://www.nice.org.uk/article/pmg9/resources/nonguidance-guide-to-the-methods-of-technologyappraisal-2013-pdf. Accessed Apr 18, 2016.

16. Moher D, Liberati A, Tetzlaff J, Altman DG, PRISMA Group. Preferred reporting items for systematic reviews and meta-analyses: the PRISMA statement. Ann Intern Med. 2009;151:264-9.

17. Downs $\mathrm{SH}$, Black N. The feasibility of creating a checklist for the assessment of the methodological quality both of randomised and non-randomised studies of health care interventions. J Epidemiol Community Health. 1998;52:377-84.

18. Hoaglin DC, Hawkins N, Jansen JP, et al. Conducting indirect-treatment-comparison and network-meta-analysis studies: report of the ISPOR Task Force on Indirect Treatment Comparisons Good Research Practices: part 2. Value Health. 2011;14:429-37.

19. Dias S, Sutton AJ, Ades AE, Welton NJ. Evidence synthesis for decision making 2: a generalized linear modeling framework for pairwise and network meta-analysis of randomized controlled trials. Med Decis Mak. 2013;33:607-17.

20. Dias S, Welton NJ, Sutton AJ, Caldwell DM, Lu G, Ades AE. Evidence synthesis for decision making 4: inconsistency in networks of evidence based on randomized controlled trials. Med Decis Mak. 2013;33:641-56.

21. Rooprai R, Nijhawan S. Comparative evaluation of renal function in patients of chronic hepatitis $B$ treated with nucleos(t)ide analogues. Hepatol Int. 2013;7:S185.

22. Esaulenko E, Stukov BV, Nikitina OE. Evaluation of patient characteristics and efficacy with entecavir and telbivudine treatment in Russian patients with chronic hepatitis B. Hepatol Int. 2013;7:S191.

23. Krastev Z, Kotzev IA, Celen MK, McNeeley D, Hamed KA. Efficacy and safety of telbivudine versus tenofovir treatment based on the Roadmap concept: results from a randomized, controlled trial in HBeAg-negative chronic hepatitis $\mathrm{B}$ patients. Hepatology. 2014;60:1104A.

24. Park H, Park JY, Kim SU, et al. A prospective randomized trial of switching to telbivudine plus adefovir in HBeAg-positive lamivudine-resistant chronic hepatitis B patients who have suboptimal response to lamivudine plus adefovir. Clin Gastroenterol Hepatol. 2014;12:157.

25. Izzedine H, Hulot JS, Launay-Vacher V, et al. Renal safety of adefovir dipivoxil in patients with chronic hepatitis B: two double-blind, randomized, placebo-controlled studies. Kidney Int. 2004;66:1153-8.

26. Lee S, Park JY, Song K, et al. Comparison of the effects of telbivudine and entecavir treatment on estimated glomerular filtration rate in patients with chronic hepatitis B. Gut Liver. 2015;9:776-83.

27. Hung $\mathrm{CH}$, Chen $\mathrm{CH}$, Lu SN, Hu TH, Wang JH, Lee $\mathrm{CM}$. Tenofovir versus entecavir in the treatment of chronic hepatitis B with severe acute exacerbation. Hepatology. 2014;60:1118A.

28. Ha NB, Ha NB, Garcia RT, et al. Renal dysfunction in chronic hepatitis B patients treated with adefovir dipivoxil. Hepatology. 2009;50:727-34.

29. Su PY, Yen HH, Hsu YC, Wu SS, Su WW, Soon MS. Renal function for chronic hepatitis B patients treated with nucleoside antivirals - a retrospective study in a medical center. Hepatol Int. 2013;7:S222.

30. Chan HLY, Chen YC, Gane EJ, et al. Randomized clinical trial: efficacy and safety of telbivudine and lamivudine in treatment-naive patients with HBV-related decompensated cirrhosis. J Viral Hepat. 2012;19:732-43.

31. Gane EJ, Deray G, Liaw YF, et al. Telbivudine improves renal function in patients with chronic hepatitis b. Gastroenterology. 2014;146(138-46):e5.

32. Koklu S, Gulsen MT, Tuna Y, et al. Differences in nephrotoxicity risk and renal effects among anti-viral therapies against hepatitis B. Aliment Pharmacol Ther. 2015;41:310-9.

33. Hu TH, Chang KC, Tseng PL, Lin MT, Hung CH, Yen YH. A comparison of renal safety of telbivudine entecavir and tenofovir treatment in chronic hepatitis B patients: a single center large "real life" cohort study. Hepatol Int. 2015;1:S186.

34. Piratvisuth T, Komolmit P, Tanwandee T, et al. 52 -Week efficacy and safety of telbivudine with conditional tenofovir intensification at week 24 in 
HBeAg-positive chronic hepatitis B. PLoS One. 2013;8:e54279.

35. Sun H, Zhang S, Wang M. Efficacy and safety of three different treatments in 183 patients with hepatitis B cirrhosis. Hepatol Int. 2014;1:S69.

36. Tsai $\mathrm{MC}$, Chen $\mathrm{CH}$, Hung $\mathrm{CH}$, et al. A comparison of efficacy and safety of 2-year telbivudine and entecavir treatment in patients with chronic hepatitis B: a match-control study. Clin Microbiol Infect. 2014;20:090-100.

37. Tsai $\mathrm{MC}$, Hung $\mathrm{CH}$, Chen $\mathrm{CH}$, et al. Comparing the change of renal function of tenofovir and telbivudine naive chronic hepatitis $\mathrm{B}$ patients. Hepatol Int. 2014;1:S154.

38. Tsai MC, Chen CH, Tseng PL, et al. Comparison of renal safety and efficacy of telbivudine, entecavir and tenofovir treatment in chronic hepatitis $\mathrm{B}$ patients: real world experience. Clin Microbiol Infect. 2016;22(95):e1-7.

39. Tien C, Xu JJ, Chan LS, et al. Long-term treatment with tenofovir in Asian-American chronic hepatitis $\mathrm{B}$ patients is associated with abnormal renal phosphate handling. Dig Dis Sci. 2015;60:566-72.

40. Qi X, Wang JY, Mao RC, Zhang JM. Impact of nucleos(t)ide analogues on the estimated glomerular filtration rate in patients with chronic hepatitis B: a prospective cohort study in China. J Viral Hepat. 2015;22:46-54.

41. Liang KH, Chen YC, Hsu CW, Chang ML, Yeh CT. Decrease of serum angiotensin converting enzyme levels upon telbivudine treatment for chronic hepatitis $B$ virus infection and negative correlations between the enzyme levels and estimated glomerular filtration rates. Hepat Mon. 2014;14:e15074.

42. Hadziyannis SJ, Tassopoulos NC, Heathcote EJ, et al. Adefovir dipivoxil for the treatment of hepatitis $\mathrm{B}$ e antigen-negative chronic hepatitis $\mathrm{B}$. N Engl J Med. 2003;348:800-7.

43. Papatheodoridis G, Buti M, Cornberg M, et al. EASL clinical practice guidelines: management of chronic hepatitis B virus infection. J Hepatol. 2012;57:167-85.

44. Wang Y, Thongsawat S, Gane EJ, et al. Efficacy and safety of continuous 4-year telbivudine treatment in patients with chronic hepatitis B. J Viral Hepat. 2013;20:e37-46.

45. Yang Q, Shi YU, Yang Y, Lou G, Lv F. Association between adefovir dipivoxil treatment and the risk of renal insufficiency in patients with chronic hepatitis B: a meta-analysis. Biomed Rep. 2015;3:269-75. 